\title{
Generalized entropic gravity from modified Unruh temperature
}

\author{
Salih Kibaroğlı* \\ Department of Physics, Kocaeli University, 41380 Kocaeli, Turkey
}

(Dated: July 9, 2019)

\begin{abstract}
In this study, the effects of the generalized uncertainty principle on the theory of gravity are analyzed. Inspired by Verlinde's entropic gravity approach and using the modified Unruh temperature, the generalized Einstein field equations with cosmological constant are obtained and corresponding conservation law is investigated. The resulting conservation law of energy-momentum tensor dictates that the generalized Einstein field equations are valid in a very limited range of accelerations. Moreover, the modified Newton's law of gravity and the modified Poisson equation are derived. In a certain limit, these modified equations reduce to their standard forms.
\end{abstract}

PACS numbers: 04.20.-q, 04.50.Kd, 04.60.-m, 95.36.+x

Keywords: Entropic gravity, extended theory of gravity, generalized uncertainty principle, quantum gravity

\section{INTRODUCTION}

In theoretical physics, one of the main studies is to generalize the theory of gravity, because we have several good reasons. For instance, merging efforts of the gravity with the standard model and the unification of quantum mechanics and Einstein's general relativity are still unclear. Within this scope, there are many methods to cope with these difficulties such as the supergravity, noncommutative geometry, quantum gravity, and string theory.

It is interesting to study the generalized theory of gravity from thermodynamic perspective. There are several theoretical studies on the thermodynamical origin of gravity. The early studies were done by Bekenstein and Hawking [1 3. They considered the formulation of the laws of black hole mechanics taking into account of the thermodynamic point of view. According to their studies, a relation has occurred between the black hole area and its entropy as $S_{B H}=A / 4 l_{p}^{2}$ which is called the Bekenstein-Hawking (BH) entropy. Their studies opened a new window to describe the emergent nature of space-time and gravity. In 1995, Jacobson was the first who claimed that the gravity may not be a fundamental interaction, but it is originated from the first law of thermodynamics on a local Rindler horizon. From this idea, he found certain components of Einstein's field equation 4. Yet another important work was achieved by Verlinde [5] (see also [6]) who formulated a thermodynamical description of gravity by considering the holographic principle and BH entropy. In this way he obtained Newton's law of gravity and Einstein's field equations. Similar to Jacobson's idea, Verlinde suggested that gravity is not a fundamental force, but emerges as an entropic force. This idea is known as the entropic gravity or emergent gravity.

The studies in the entropic gravity proposal can be divided into two approaches [7]. The first one is the thermodynamic gravity (TG) which is used to obtain the Einstein field equation from the BH entropy [4 6, 8, 10, and the second one is the holographic gravity (HG) in which the Einstein's equation can be obtained by keeping entropy stationary in equilibrium under the variations of space-time geometry and the quantum states within a small region [1]-13.

In the light of the above theoretical background, it is easy to say that the entropic gravity approach provides a powerful framework to generalize the theory of gravity. For instance, if we use generalized thermodynamic quantities such as entropy in the entropic gravity approach, we can find a generalized model of the theory of gravity [1421. Therefore, it may help us to find a deep understanding of the nature of space-time and gravity. This idea motivated us to study the relation between the generalized uncertainty principle (GUP) and entropic gravity. For this purpose, we consider the GUP corrected Unruh temperature to modify the theory of gravitation. Therefore, using Verlinde's entropic gravity approach [5] and the modified Unruh temperature [22, we derive the generalized Einstein field equations with the cosmological term and analyze corresponding conservation law. We also find GUP corrected Newton's law of gravity and Poisson's equation.

The paper is organized as follows. In Sec. 2, the historical background for the Unruh effect and the modification of Unruh temperature based on GUP are shortly reviewed. In Sec. 3, the generalized Einstein's field equations are obtained. In Sec. 4, we find the generalized forms of Newton's law of gravity and Poisson's equation. The last section is devoted to the discussion of the obtained results.

\footnotetext{
*salihkibaroglu@gmail.com
} 


\section{GUP CORRECTED UNRUH TEMPERATURE}

Discovery of the Hawking effect [2, 3] demonstrates that black holes should radiate with a temperature $T_{H}=$ $\hbar g / 2 \pi k_{B} c$ where $g$ is the gravitational acceleration, $k_{B}$ is Boltzman's constant and $c$ is the speed of light. The Hawking effect also describes the black-body radiation which is produced by the vacuum fluctuations near the event horizon of the black hole. Moreover, there is another approach which is so-called the Unruh effect (or the FullingDavies-Unruh effect) [23 25]. The Unruh effect describes the motion of an accelerated observer in a flat Minkowski spacetime. In this case, the observer observes black-body radiation released from the Rindler horizon and experiences following temperature,

$$
T_{U}=\frac{\hbar a}{2 \pi k_{B} c}
$$

as though it is in a thermal field where an inertial observer would observe none. Here, $a$ is the acceleration of the reference frame. This temperature is called the Unruh temperature (or the Hawking-Unruh temperature). The Unruh effect, similar to the Hawking effect, is originated from the vacuum fluctuations which cause a particle-antiparticle pairs creation near the Rindler horizon of the accelerated frame. This effect is important to understand the notion of particle emission from cosmological horizons and black holes (for more detail see [26, 27]).

The formulation of the Unruh effect can be obtained by using the Heisenberg uncertainty principle (HUP) when we take into account of a photon that has crossed the Rindler event horizon. In this case, the position uncertainty of the photon depends on the crossing point of the Rindler event horizon. In this framework, a direct derivation of the Unruh effect with the help of HUP can be found in [28] (see also [29]).

We know from early studies 30,42 that HUP should be generalized when we consider the Planck scale where the gravitational effect is neglected. So, we can say that such a generalization may lead to modify the Unruh effect. In this case, we can use the generalized uncertainty principle (GUP) which provides the determination of the quantum gravitational corrections for HUP. This approximation is used in various branches of physics such as the string theory, loop quantum gravity, and non-commutative quantum mechanics (for more detail see review [43]). For instance, GUP is used to find quantum gravitational corrections for the Planck-scale black hole thermodynamics including the Unruh temperature [22] and the Hawking temperature [44. Now, we shall briefly review the paper [22] which includes the GUP corrected Unruh temperature. In order to obtain a generalized version of the Unruh temperature, the authors used the following form of GUP,

$$
\Delta x \Delta p \geq \frac{\hbar}{2}\left[1+\beta\left(\frac{\Delta p}{m_{p}}\right)^{2}\right], \quad[\hat{x}, \hat{p}]=i \hbar\left[1+\beta\left(\frac{\hat{p}}{m_{p}}\right)^{2}\right]
$$

where the constant $\beta$ is a dimensionless deformation parameter and the other constants are chosen as $c=1, k_{B}=1$ throughout this paper. Considering this GUP background and using the quantum field theoretic calculations, the generalized Unruh temperature can be described to the first approximation in $\beta$,

$$
T \bumpeq T_{U}\left[1+\beta \pi \Omega\left(\frac{l_{p} a}{\pi}\right)^{2}\right],
$$

where $\Omega$ is the Rindler frequency and $l_{p}=\sqrt{\hbar G / c^{3}} \simeq 10^{-35} \mathrm{~m}$ is the Planck length. In a certain condition, we can take the Rindler frequency as $\Omega \approx 1 / 2 \pi$, and then Eq. (3) takes following form [22],

$$
T \bumpeq T_{U}\left[1+\frac{\beta}{2}\left(\frac{l_{p} a}{\pi}\right)^{2}\right]=T_{U}\left[1+\frac{\beta}{2}\left(\frac{T_{U}}{m_{p}}\right)^{2}\right] .
$$

Thus, taking account of the Eq.(1), the temperature $T$ is obtained with a dependence of the cubic power of the acceleration. We can also write the last equations in a simple form,

$$
T=T_{U} f(a)=\frac{\hbar a}{2 \pi} f(a)
$$

where the function $f(a)$ is defined by, 


$$
f(a)=\left[1+\frac{\beta}{2}\left(\frac{l_{p} a}{\pi}\right)^{2}\right]=\left[1+\frac{\beta}{2}\left(\frac{T_{U}}{m_{p}}\right)^{2}\right],
$$

where $m_{p}=\hbar / 2 l_{p} \simeq 10^{-8} \mathrm{~kg}$ is the Planck mass. This correction function tends to increase the Unruh temperature for selected GUP model. Different kind of $f(a)$ functions can also be found in the literature (for the maximal acceleration principle [45, 46] or the extended uncertainty principle [47]). Setting the deformation parameter as $\beta=0$, it is easy to see that Eq. 44 reduces to the standard Unruh temperature.

\section{GENERALIZED EINSTEIN FIELD EQUATIONS}

The thermodynamic origin of gravity, also known as the entropic gravity, is a theory that describes the gravitational force by using thermodynamic quantities such as energy, temperature, and entropy. Thus, if one modifies the mentioned quantities, the gravity should be modified. In the study [18, Wang unified several modified entropic gravity models by inserting a model dependent factor $f(a, A)$ into the equipartition rule $E=\frac{a A}{4 \pi G} f(a, A)$. In this idea, he found modified Einstein's and Newton's gravitational field equation by considering $E$ as total energy in a closed holographic surface. Here, $a$ corresponds to the (redshifted) surface acceleration and $A$ is the area of the holographic surface. In this section, we will follow Verlinde's entropic gravity proposal 5 and Wang's method to find quantum corrected Einstein's field equations by using the GUP corrected Unruh temperature in Eq.(5). In analogy to Wang's study, we will use $f(a)$ in Eq. (6) as the model dependent correction factor.

Verlinde considers the black hole horizon as a spherically symmetric holographic screen $\mathcal{S}$ based on Bekenstein's idea [1. In this consideration, we have a test particle with mass $m$ and an acceleration $a$ moving along to the screen (see Figure 11). To find the relativistic gravitational field equation, we consider a static background with a global time-like Killing vector $\xi^{a}$ which is normalized as $\xi^{a} \xi_{a}=-e^{2 \phi}$. Here, $\phi$ represents the natural generalization of Newton's potential in general relativity [48] which can be written as

$$
\phi=\frac{1}{2} \ln \left(-\xi^{a} \xi_{a}\right)
$$

We also note that the exponential of Eq.(7) represents the redshift factor that relates the local time coordinate at a reference point with $\phi=0$.

In our case, we will use $\phi$ to describe a foliation of space and consider our holographic screen at surfaces of constant redshift. Because the entire screen uses the same time coordinate (for more details see [5]). Let us consider a force that acts on the particle of mass $m$. To obtain the emergence of inertia and the equivalence principle, we have to relate this Killing vector field with the temperature and the entropy gradients. In this framework, we want to show that the usual geodesic motion of particles can be considered as being the result of an entropic force. The four-velocity $u^{b}$ and its four-acceleration $a^{b}$ of the particle can be expressed by using the Killing vector field as,

$$
u^{b}=e^{-\phi} \xi^{b}, \quad a^{b}=e^{-2 \phi} \xi^{a} \nabla_{a} \xi^{b} .
$$

With the help of the Killing equation $\nabla_{a} \xi_{b}+\nabla_{b} \xi_{a}=0$ and Eq. (7), the four-acceleration can be defined in terms of the potential $\phi$ as,

$$
a^{b}=-\nabla^{b} \phi
$$

Here, the potential $\phi$ is just used as a device which satisfies the relation in Eq. (7) to define the acceleration. Moreover, the acceleration is perpendicular to the screen, so we can write the acceleration as a scalar quantity by contracting it with a unit outward pointing vector $N^{a}$ which is normal to both the holographic surface $\mathcal{S}$ and the Killing vector $\xi^{a}$. From these definitions, the local temperature $T$ on the holographic screen is defined in analogy to Eq. (5),

$$
T=\frac{\hbar}{2 \pi} f(\nabla \phi) e^{\phi} N^{a} \nabla_{a} \phi
$$

where the GUP correction function transformed as $f(a) \rightarrow f(\nabla \phi)$ due to the definition of the acceleration in Eq. (9p). Here, we used the exponential $e^{\phi}$ as a redshift factor, because the temperature is measured with respect to the reference point at infinity [5]. 


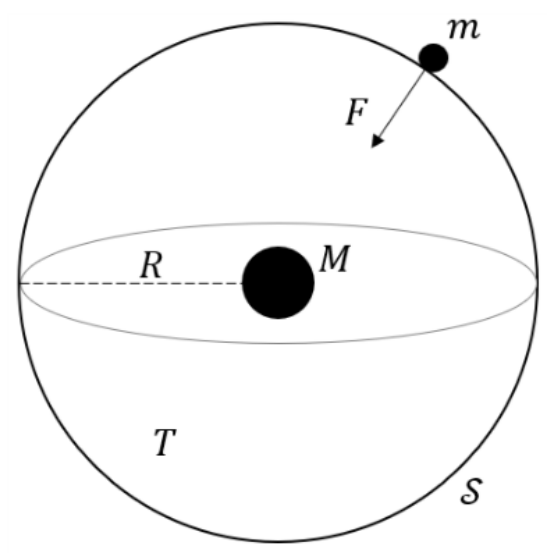

Figure 1. Suppose that we have two masses, one is a test particle with mass $m$ close to spherical holographic screen $\mathcal{S}$, and the other is the source with mass $M$ surrounded by the screen.

To find the force that acts on a particle which is very close to the screen, we first consider the change in entropy at the screen is $2 \pi$ for a displacement by one Compton wavelength normal to the screen,

$$
\nabla_{a} S=-2 \pi \frac{m}{\hbar} N_{a}
$$

So, one can obtain the entropic force by using the last equation and Eq.10] as follows,

$$
F_{a}=T \nabla_{a} S=-m e^{\phi} f(\nabla \phi) \nabla_{a} \phi .
$$

This is the GUP corrected model of the relativistic analogue of Newton's second law of gravity. It defines the gravitational force that acts on the particle which located close to the screen $\mathcal{S}$. If we take the limit of low acceleration and low potential where the redshift factor can be ignored, then Eq. 12 reduces its non-relativistic form $F=$ maf $(a)$.

Now, we would like to find a generalized version of Einstein's field equation by combining Verlinde's entropic gravity proposal with the GUP model in Eq.(2). According to Bekenstein, if there is a test particle which closes to the black hole horizon a one Compton wavelength, the test particle increases the mass and horizon area of the black hole. This process is defined as one bit of information. Considering the holographic screen on a closed surface with constant redshift, the total number of bits $N$ on the screen can be specified as,

$$
N=\frac{A}{G \hbar}
$$

where $A$ is the area of closed surface on the screen and $G$ is a constant which will be identified as the Newton's gravitational constant. In this case, we assume that the energy which is related to source mass $M$ is distributed over all the bits. According to the equipartition law of energy $E=\frac{1}{2} T N$, and using the relation $E=M$, the total mass can be written as

$$
M=\frac{1}{2} \oint_{\mathcal{S}} T d N
$$

Substituting the Eq. 10 and Eq. 13 in Eq. 14 , we get,

$$
M=\frac{1}{4 \pi G} \oint_{\mathcal{S}} f(\nabla \phi) e^{\phi} \nabla \phi d A .
$$

This equation can be seen as the natural generalization of Gauss' law to the General Relativity and $M$ corresponds to the Komar mass. Here, the differential surface element is described by,

$$
|d A|=d x^{a} \wedge d x^{b} \epsilon_{a b c d} e^{-\phi} \xi^{c} N^{d} .
$$


Thus, the Komar mass can be written as follows by using the Stokes' theorem, Eq. [7] and $\nabla_{a} \nabla^{a} \xi^{b}=-R_{a}^{b} \xi^{a}[16$, 18],

$$
M=\frac{1}{4 \pi G} \int_{\mathcal{V}}\left\{R_{a b} \xi^{b} n^{a}+\left[\nabla_{b} f(a)\right]\left[\nabla_{a} \xi^{b}\right] n^{a}\right\} d V
$$

where $R_{a b}$ is the Ricci curvature tensor, $\mathcal{V}$ is the three dimensional volume bounded by the holographic screen and $n^{a}$ is its outward normal. For simplicity, we used $f(a)$ rather than $f(\nabla \phi)$. Due to the relation $\xi^{b} \nabla_{b} f(a)=0$ which is demonstrated in [18, the second term in the parenthesis can be written by,

$$
\left[\nabla_{b} f(a)\right]\left[\nabla_{a} \xi^{b}\right] n^{a}=-n^{a} \xi^{b} \nabla_{a} \nabla_{b} f(a)
$$

The Komar mass can also be written as an integral over the enclosed volume of certain components of the energymomentum tensor $T_{a b}$ [4] as,

$$
M=2 \int_{\mathcal{V}}\left(T_{a b}-\frac{1}{2} g_{a b} T\right) \xi^{b} n^{a} d V
$$

where $g_{a b}$ is the spacetime metric tensor. If we compare Eqs. (17) and (19), we obtain the generalized Einstein field equations as follows,

$$
f(a) R_{a b}-\nabla_{a} \nabla_{b} f(a)=8 \pi G\left(T_{a b}-\frac{1}{2} g_{a b} T\right) .
$$

This is only a time-time component of the field equation [5]. The last expression can also be written as,

$$
R_{a b}-\frac{1}{2} g_{a b} R-\frac{1}{f(a)}\left(\nabla_{a} \nabla_{b}-\frac{1}{2} g_{a b} \nabla^{2}\right) f(a)=\frac{8 \pi G}{f(a)} T_{a b}
$$

and in a simple form,

$$
R_{a b}-\frac{1}{2} g_{a b} R=\frac{8 \pi G}{f(a)}\left\{T_{a b}+T_{a b}(a)\right\} .
$$

The last result can be seen as the GUP corrected Einstein field equations with a new additional energy-momentum tensor as a function of the acceleration which is defined,

$$
T_{a b}(a)=\frac{1}{8 \pi G}\left(\nabla_{a} \nabla_{b} f(a)-\frac{1}{2} g_{a b} \nabla^{2} f(a)\right) .
$$

Due to this new contribution, the energy momentum conservation law should be revisited. Considering the usual form of Einstein's field equations $G_{b}^{a}=8 \pi G T_{b}^{a}$, the covariant conservation law of the energy-momentum tensor can be written as $\nabla_{a} T_{b}^{a}=0$, and in accordance with this result the covariant derivative of the Einstein tensor $G_{b}^{a}$ goes to zero,

$$
\nabla_{a}\left(R_{b}^{a}-\frac{1}{2} \delta_{b}^{a} R\right)=0 .
$$

In our case, the gravitational field equation in Eq. 21 is more complicated than the standard one. In order to determine corresponding conservation law, we take the covariant derivative of Eq.21, then we get the following equations,

$$
R \nabla_{a} f(a)+\nabla_{a} \nabla^{2} f(a)=-16 \pi G \nabla_{a} T_{b}^{a}
$$

The right-hand side of the last equation should be zero because of the well-known relation $\nabla_{a} T_{b}^{a}=0$, so we find following constraint,

$$
R \nabla_{a} f(a)=-\nabla_{a} \nabla^{2} f(a)
$$


As a consequence of the last equation, one can say that the energy momentum tensor is conserved only if the constraint is satisfied. In other words, it can be seen as a restriction for this kind of modified entropic gravity because only few values for $a^{b}=-\nabla^{b} \phi$ are permitted by this requirement. According to Wang's paper [18, this result reminds the Chern-Simons (CS) modification of general relativity [49 in which conservation of the energy-momentum is unwarranted by gravitational equations. So, one can consider the last equation as a criterion of admissible metrics in modified models of entropic gravity. In the limit of $\beta=0$, we therefore recover the standard Einstein field equation,

$$
R_{a b}-\frac{1}{2} g_{a b} R=8 \pi G T_{a b} .
$$

Yet another alternative is to consider Komar mass formula with nonzero cosmological constant $\Lambda$ [15, 48],

$$
M=2 \int_{\mathcal{V}}\left(T_{a b}-\frac{1}{2} g_{a b} T+\frac{\Lambda}{8 \pi G} g_{a b}\right) n^{a} \xi^{b} d V
$$

If we compare the last equation with Eq.(17), we get the following field equations,

$$
R_{a b}-\frac{1}{2} g_{a b} R+\frac{\Lambda}{f(a)} g_{a b}=8 \pi \frac{G}{f(a)}\left\{T_{a b}+T_{a b}(a)\right\},
$$

which is the generalized Einstein field equation with scaled cosmological constant. In addition, according to Eq. (29), the constraint equation in Eq. 26 remains unchanged because the covariant derivative of cosmological constant is zero.

\section{MODIFIED NEWTON'S LAW OF GRAVITY AND POISSON'S EQUATION}

Suppose that a test particle of mass $m$ moves a distance $\Delta x$ orthogonal to the holographic screen as in Figure 2 . In this case, there is a relation between the change of entropy $\Delta S$ and the distance of the particle from screen $\Delta x$ [ ,

$$
\Delta S=2 \pi \frac{m}{\hbar} \Delta x
$$

The last equation demonstrates that the entropy is proportional to the information of the test particle and it can be seen as one of the main formulas for construction of the entropic force. The change of entropy given in Eq. (30) can also be written as $\Delta S=2 \pi \Delta x / \lambda_{m}$ by using the definition of the Compton wavelength $\lambda_{m}=\hbar / m$. In the case of $\Delta x \simeq \lambda_{m}$, the particle is absorbed by the screen and this event leads to increase the entropy of the system [5]. According to the Verlinde's proposal, the entropic force is defined as follows,

$$
F=T \frac{\Delta S}{\Delta x},
$$

where $T=T_{0} f(a)=\frac{\hbar a}{2 \pi} f(a)$ represents the temperature which is defined in analogue to Eq. (5). Here, $a$ represents the acceleration of the particle with mass $m$. Substituting the temperature and Eq. 30. in Eq.(31), we get the gravitational force acting on a particle of mass $m$ as,

$$
F=m a f(a)=m a\left[1+\frac{\beta}{2}\left(\frac{l_{p} a}{\pi}\right)^{2}\right],
$$

or it can alternatively be written in terms of $T_{0}$ by using Eq.(6),

$$
F=m a\left[1+\frac{\beta}{2}\left(\frac{T_{0}}{m_{p}}\right)^{2}\right] .
$$

The Eqs. (32) and (33) represent the modification of Newton's second law of gravity, $F=m a$, with the Planck scale correction based on the GUP model in Eq. (2). This result is consistent with the non-relativistic analogue to Eq. (12) which is found in the previous section. Similar modifications can be found in 5054 . 


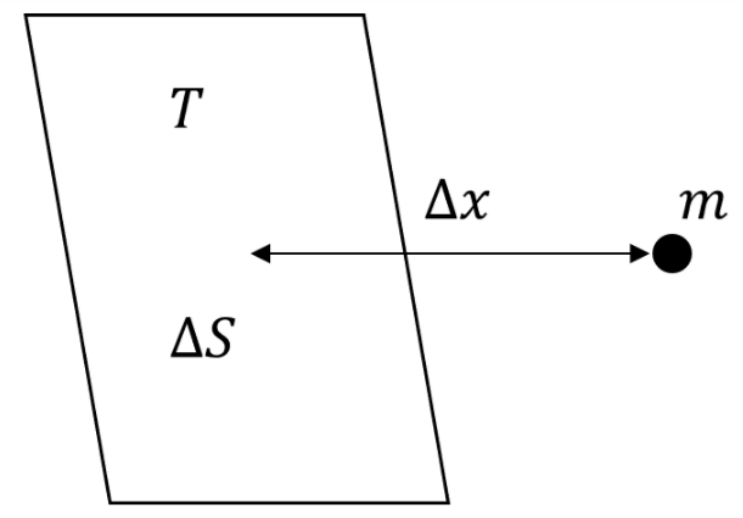

Figure 2. A particle with mass $m$ is moving to a part of the holographic surface. The screen which corresponds to emerged part of space contains the particle and stores data which describes the part of the space that has not yet emerged [5].

Now we want to determine the effects of the GUP model on Poisson's equation. For this purpose, we use a holographic screen $\mathcal{S}$ which corresponds to an equipotential surface with fixed Newton potential $\Phi_{0}$. We will assume that the volume which enclosed by the screen contains the mass distribution given by $\rho(\vec{r})$, and all test particles are outside of this volume. To define the force on the particles, we need to identify the temperature [5]. Similar to the Unruh temperature in Eq.(1), we first define the acceleration. This acceleration can be found by considering a test particle and moving it close to the screen. The local acceleration of the test particle can be defined by a potential $\Phi$ as,

$$
\vec{a}=-\vec{\nabla} \Phi
$$

where the potential $\Phi$ is just used to a variable to describe the local acceleration, but we don't know yet it has a relation with the mass distribution. In this condition, the correction factor can be viewed as $f(a) \rightarrow f(\vec{\nabla} \Phi)$. So, the local temperature $T$ in analogue with Eq.(5) can be defined as

$$
T=\frac{\hbar}{2 \pi} f(\vec{\nabla} \Phi)|\vec{\nabla} \Phi|
$$

Substituting the last equation into Eq.(14) and using Eq.(13) we get,

$$
M=\frac{1}{4 \pi G} \oint_{\mathcal{S}} f(\vec{\nabla} \Phi) \vec{\nabla} \Phi \cdot d \vec{A}
$$

This result can be written by using the divergence theorem as follows,

$$
M=\frac{1}{4 \pi G} \int_{\mathcal{V}} \vec{\nabla} \cdot[f(\vec{\nabla} \Phi) \vec{\nabla} \Phi] d V,
$$

where $\mathcal{V}$ represents three dimensional volume element. The mass distribution of the volume in the closed surface $\mathcal{S}$ can also be given as

$$
M=\int_{\mathcal{V}} \rho(\vec{r}) d V .
$$

Comparing Eq.37] with Eq. 38], we get following equation which contains a relation between the potential and the mass distribution,

$$
\vec{\nabla} \cdot[f(\vec{\nabla} \Phi) \vec{\nabla} \Phi]=4 \pi G \rho(\vec{r})
$$


If one takes $f(\vec{\nabla} \Phi)=1$, then the potential $\Phi$ satisfies the usual Poisson's equation as follows

$$
\nabla^{2} \Phi=4 \pi G \rho(\vec{r})
$$

Therefore, Eq. $\sqrt{39}$ ) can be interpreted as the quantum corrected Poisson's equation and this result shows that there is an exact contribution to the Poisson equation comes from GUP (for similar modification of Poisson's equation see [16, 55, 56]).

\section{CONCLUSION}

In this work, we investigated the possible effects of generalized uncertainty principle (GUP) on Einstein's theory of general relativity and Newton's law of gravity. For this aim, we considered Verlinde's entropic gravity proposal and the GUP corrected Unruh temperature. Using these approaches, we derived generalized version of the Einstein field equations with scaled cosmological constant and an additional energy-momentum tensor $T_{a b}(a)$ by using Wang's perspective [18. The additional energy-momentum tensor in Eq. 29) dictates that the modified temperature gives rise to a new source term in Einstein's field equation. This kind of source term may be related to the dark energy [57, 58. For this reason, the cosmological consequences of the Eq. 22 and Eq. 29p are open problems. Besides, if one consider the $f(R)$ theories of gravity (for more detail see review [59]), the modified Einstein's field equation can be given as,

$$
R_{a b}-\frac{1}{2}\left[\frac{f(R)}{\partial_{R} f(R)}\right] g_{a b}-\frac{1}{\partial_{R} f(R)}\left(\nabla_{a} \nabla_{b}-g_{a b} \nabla^{2}\right) \partial_{R} f(R)=\frac{8 \pi G}{\partial_{R} f(R)} T_{a b}
$$

where $f(R)$ is a function of the Ricci scalar $R, \partial_{R}$ is the derivative with respect to $R$ and $T_{a b}$ is the energy-momentum tensor of the matter fields. In our context, if one take the function as $f(R)=f(a) R$ by the help of Eq.(6), there is an exact similarity arises between the last equation and the GUP corrected Einstein's field equation in Eq. 21). In addition, it is well-known that the equivalence between the $f(R)$ theory and the scalar-tensor theories, so one may find different relations between $f(R)$ and $f(a)$ in the framework of the Brans-Dicke theory or the conformal gravity.

We also analyzed the energy-momentum conservation law of the generalized Einstein field equations and found a constraint Eq.26] which is required to satisfy corresponding conservation law. As a consequence of this constraint, according to Wang [18, the modifications of Einstein's equation in Eq.29] are valid only in very restricted conditions since only a few values for $a^{b}$ are allowed by this constraint.

The relativistic and nonrelativistic forms of Newton's law of gravity were obtained with GUP corrections in Eqs. $(12)$ and $(32)$ respectively. These modifications have similar prediction with the Randall-Sundrum II model 60 which includes one uncompactified extra dimension. Parallel results for different models of GUP can be found in [61. Also, the GUP corrected Poisson equation was found by using the entropic gravity approximation in Eq.(39).

The deformation parameter $\beta$ was studied for the gravitational and non-gravitational regimes in the literature (the corresponding results was reviewed in 62]). For the gravitational regime, the deformation parameter should be $\beta<10^{69}$ for the perihelion precession (Solar system data) in gravitational measurements. According to the paper 44, the parameter was also calculated as $\beta=82 \pi / 5$ in the context of the quantum field theory and the general theory of relativity. Taking the consideration of the non-gravitational case, the parameter takes values of $\beta<10^{20}$ and $\beta<10^{46}$, respectively, for the Lamb shift and the Landau levels. Our results may provide a new background to study the mentioned areas 62] for the gravitational regime. Besides, it is easy to show that, in a certain limit $(\beta=0)$, the results reduce to their conventional forms.

Our results and the papers [14 21] show that the entropic gravity proposal provides a powerful framework to obtain the generalized theory of gravity by using thermodynamical quantities.

\section{ACKNOWLEDGMENTS}

The author would like to thank Oktay Cebecioğlu and Mustafa Senay for valuable discussions.

[1] J. D. Bekenstein, Phys. Rev. D 7, 2333 (1973).

[2] S. W. Hawking, Nature 248, 30 (1974). 
[3] S. W. Hawking, Commun. Math. Phys. 43, 199 (1975).

[4] T. Jacobson, Phys. Rev. Lett. 75, 1260 (1995).

[5] E. Verlinde, J. High Energy Phys. 04, 029 (2011).

[6] E. Verlinde, SciPost Phys. 2, 016 (2017).

[7] S. M. Carroll, G. N. Remmen, Phys. Rev. D 93, 124052 (2016).

[8] R. G. Cai, S. P. Kim, JHEP 02, 050 (2005).

[9] T. Padmanabhan, Rept. Prog. Phys. 73, 046901 (2010).

[10] Y. Tian, X. N. Wu, Phys. Rev. D 81, 104013 (2010).

[11] T. Faulkner, M. Guica, T. Hartman, R. C. Myers, M. van Raamsdonk, JHEP 1403, 051 (2014).

[12] N. Lashkari, M. B. McDermott, M. Van Raamsdonk, JHEP 04, 195 (2014).

[13] T. Jacobson, Phys. Rev. Lett. 116, 201101 (2016).

[14] R. G. Cai, L. M. Cao, N. Ohta, Phys. Rev. D 81, 061501(R) (2010).

[15] C. M. Ho, D. Minic, Y. J. Ng, Phys. Lett. B 693, 567 (2010).

[16] A. Sheykhi, S. K. Rezazadeh, JCAP 1210, 012 (2012).

[17] S. H. Hendi, A. Sheykhi, Int. J. Theor. Phys. 51, 1125 (2012).

[18] T. Wang, Sci. China-Phys. Mech. Astron., 57, 1623-1629 (2014).

[19] H. Moradpour, A. Sheykhi, Int. J. Theor. Phys. 55, 1145-1155 (2016).

[20] E. Dil, Can. J. Phys. 93, 1274 (2015).

[21] M. Senay, S. Kibaroğlu, Int. J. Mod. Phys. A 33, 1850218 (2018).

[22] F. Scardigli, M. Blasone, G Luciano, R. Casadio, Eur. Phys. J. C 78, 728 (2018).

[23] S. A. Fulling, Phys. Rev. D 7, 2850 (1973).

[24] P. C. W. Davies, J. Phys. A 8, 609 (1975).

[25] W. G. Unruh, Phys. Rev. D 14, 870 (1976).

[26] P. M. Alsing, P. W. Milonni, American Journal of Physics 72, 1524 (2004).

[27] L. C. B. Crispino, A. Higuchi, G. E. A. Matsas, Rev. Mod. Phys. 80, 787 (2008).

[28] F. Scardigli, Nuovo Cim. B 110, 1029 (1995).

[29] J. Giné, EPL 121, 10001 (2018).

[30] H. S. Snyder, Phys. Rev. 71, 38 (1947).

[31] C. N. Yang, Phys. Rev. 72, 874 (1947).

[32] C. A. Mead, Phys. Rev. 135, B849 (1964).

[33] F. Karolyhazy, Nuovo Cim. A 42, 390 (1966).

[34] D. Amati, M. Ciafaloni, G. Veneziano, Phys. Lett. B 197, 81 (1987).

[35] D. Amati, M. Ciafaloni, G. Veneziano, Int. J. Mod. Phys. A 3, 1615 (1988).

[36] D. J. Gross, P. F. Mende, Nucl. Phys. B 303, 407 (1988).

[37] M. Maggiore, Phys. Lett. B 304, 65 (1993).

[38] Y. J. Ng and H. van Dam, Mod. Phys. Lett. A 9, 335 (1994).

[39] A. Kempf, G. Mangano, R. B. Mann, Phys. Rev. D 52, 1108 (1995).

[40] C. Rovelli, Phys. Rev. Lett. 77, 3288 (1996).

[41] F. Scardigli, Phys. Lett. B 452, 39 (1999).

[42] R. J. Adler, D. I. Santiago, Mod. Phys. Lett. A 14, 1371 (1999).

[43] A. N. Tawfik, A. M. Diab, Rep. Prog. Phys. 78, 126001 (2015).

[44] F. Scardigli, G. Lambiase, E. C. Vagenas, Phys. Lett. B 767, 242-246 (2017).

[45] E. Benedetto, A. Feoli, Mod. Phys. Lett. A 30, 1550075 (2015).

[46] G. G. Luciano, L. Petruzziello, Eur. Phys. J. C 79, 283 (2019).

[47] W. S. Chung, H. Hassanabadi, Phys. Lett. B 793, 451-456 (2019).

[48] R. M. Wald, General Relativity, (The University of Chicago Press, Chicago, 1984).

[49] R. Jackiw, S. Y. Pi, Phys. Rev. D 68, 104012 (2003).

[50] S. Gosh, arXiv:1003.0285 3 [hep-th].

[51] K. Nozari, P. Pedram, M. Molkara, Int. J. Theor. Phys. 51, 1268-1275 (2012).

[52] M. A. Santos, I. V. Vancea, Mod. Phys. Lett. A 27, 1250012 (2012).

[53] H. Moradpour, A. Sheykhi, C. Corda, I. G. Salako, Phys. Lett. B 783, 82-85 (2018).

[54] B. Bagchi, A. Fring, Int. J. Mod. Phys. B 33, 1950018 (2019).

[55] P. V. Pikhitsa, arXiv:1010.0318v3 [astro-ph.CO].

[56] C. Zhe, L. Ming-Hua, L. Xin, Commun. Theor. Phys. 56, 184-192 (2011).

[57] J. Frieman, M. Turner, D. Huterer, Ann. Rev. Astron. Astrophys. 46, 385 (2008).

[58] T. Padmanabhan, Adv. Sci. Lett. 2, 174 (2009).

[59] A. De Felice, S. Tsujikawa, Living Rev. Relativity 13, 3 (2010).

[60] L. Randall, R. Sundrum, Phys. Rev. Lett. 83, 4690 (1999).

[61] A. Awad, A. F. Ali, Cent. Eur. J. Phys. 12, 245-255 (2014).

[62] G. Lambiase, F. Scardigli, Phys. Rev. D 97, 075003 (2018). 